\title{
New Conditions of The Existence of Fixed Point in $\Delta$ - Ordered Banach Algebra
}

\author{
Boushra Youssif Hussein \\ Department of Mathematics, College of Education, University of AL-Qadisiyah \\ Boushra.alshebani@qu.edu.iq
}

\begin{abstract}
The main idea is to construct a new algebra and find new necessary and sufficient conditions equivalent to the existence of fixed point. In this work, an algebra is constructed, called $\Delta$ - ordered Banach algebra, we define convergent in this new space, Topological structure on $\Delta$ - ordered Banach Algebra and prove this as Housdorff space. Also, we define new conditions as $\Delta-$ lipshtiz,$\Delta-$ contraction conditions in this algebra construct, we prove this condition is the existence and uniqueness results of the fixed point. In this paper, we prove a common fixed point if the self-functions satisfy the new condition which is called $\varphi$-contraction .
\end{abstract}

Keywords: Fixed point, Ordered Banach algebra, lipschitz mapping, and contraction mappimg

\section{Introduction}

It is known Banach contraction principle and a number of generality in background of metric spaces play a fundamental role for several complications of functional analysis, differential and integral equations.

Gahler (1963) [ 6] presented the notion of 2-metric spaces as a generalization of an usual metric space. Gahler proved that geometrically $d(a, b, c)$ represents the region of a triangle formed by the point $a, b, c \in X$ equally its vertices.

An usual metric space is a continuous function, but $\mathrm{Ha}$, Cho and While (1988) [15] examined that a 2- metric space is not a continuous mapping. Dhage (1984) [5] introduced the notion of a $D$ - metric space as a generality of a 2-metric space; and studied the topological properties of $D$ - metric space

Mustafa and Sim (2006) [17] introduced a newfangled metric called $G$-metric space. They show the topological constructions of Dhages [4] work unacceptable, after Sedghi, Shobe and Zhom (2007) [20] presented concept, which is named $D^{*}$ - metric space, but Fernardcz ,Sle, Saxena, Malviya and Kuman (2017)[13] generalized an $S$ metric space to $A$-metric space.

Many researchers have their consideration to generalizing mertic (see Yan and Shao Yuan on (2011) [25], Sastry, Srinivas, Chandra and Balaiah (2011) [14], Kim and Soo (2012)[20], Dey and Saha (2013) [4],

Liu and Xu (2013) [8] introduced some concepts of a cone metric space over Banach algebra. Some researchers then developed many concepts as, Nashine and Altun, (2012)[9], Tiwari and Dubey (2013) [22], Arun and Zaheer (2014) [3]. But Nashine and Altun (2012) [10] defined cone metric spaces and proved some fixed point theorems of contractive maps in such a space using the normality condition. Also, Rahimi \& Soleimani (2014) [12] used the notion ordered cone metric space.

But some scholars have attention about fixed point theorem such as Badshah, Bhagatand and Shukla(2016)[23] how introduced some fixed point theorem for $\alpha$ - $\phi$ - metric mapping in 2- metric spaces and Ma, Jiang and Hongkaisun (2014) [24] state fixed point theorem on $C^{*}$-algebra valued metric spaces.

The point $x$ - that satisfies the equation $x=T(x)$ is called a fixed point of the function $T$ which is considered the root of the equation above. To find this root, we first find an initial holding value of $x_{0}$. Then, we calculate the value of the function $T$ in $x_{0}$ to get another root called $x_{1}$ that is $x_{1}=T\left(x_{0}\right)$; and then repeat the process can get 
a new approximate value $x_{2}=T\left(x_{1}\right)$. Thus, a sequence of root values can be generated by applying the formula $x_{n+1}=T\left(x_{n}\right)$ for $n=0.1,2, \ldots$

The fixed point a in the equation above represents the distance of the intersection point of the curves of $y=x$, $y=T(x)$ for each axis $x_{1} y$. If $x_{0}$ is the initial fixed point, then $T\left(x_{0}\right)$ is the length of the column from $x_{0}$ on the $x$ axis until it intersects the curve of the $T$ - function and since the points on the rectangle $y=x$ are equal to the distance from both axes $y$ and $x$, so the line passing at the point $\left(x_{0}, T\left(x_{0}\right)\right)$ rectangle the $x$-axis will intersect the line $y=x$ in the $x$-axis, represent $x_{1}$ where

$$
\mathrm{x}_{1}=\mathrm{T}\left(\mathrm{x}_{0}\right)
$$

In a similar way, we find the remaining points where $x_{n+1}=T\left(x_{n}\right)$. Here, we ask the following question: How do we choose the function $T$ to ensure that the generated values are converged from the repeated formula $x_{n+1}=$ $\mathrm{T}\left(\mathrm{x}_{\mathrm{n}}\right)$ ?

To answer the question, we can prove the existence and uniqueness of fixed point under some new conditions by constructing a new algebra called $\Delta-$ ordered Banach algebra .

\section{1- $\Delta$ - Ordered Banach Algebra}

We start this section by a definition of Banach algebra.

"Definition (2.1)[2] :let $E$ is a linear space over field of real numbers $E$ is called Banach algebra if $E$ is Banach space with an operation of multiplication is defined as following :for $x, y, z \in A$, for all $\alpha \in R$

1) $(x y) z=x(y z)$

2) $x(y+z)=x y+x z$ and $(x+y) z=x z+y z$

3) $\alpha(x y)=(\alpha x) y=x(\alpha y)$

4) $\|x y\| \leq\|x\|\|y\|$

We consider a Banach algebra has an identity, that is $e x=x e$ for all $x \in E$. (Multiplicative identity)

If there is an element $y \epsilon A$ such that $=y x=e, y \epsilon E$ is called inverse of $x$ and denoted by $x^{-1} . "$

"Proposition 2.2 [19]: Let $E$ be Banach algebra has a unite $e, x \in E$. If the condition spectral radius $\sigma_{\epsilon}(x)<1($ for all $\varepsilon>0)$, then

$$
(e-x)^{-1}=\sum_{i=0}^{\infty} a^{i}
$$

"Remark 2.3 [19]: Let $E$ be Banach algebra with spectral radius $\sigma_{\epsilon}(x)$ of $x$ satisfy $\sigma_{\epsilon}(x) \leq\|x\| . "$

"Remark 2.4 [2]: If $\sigma_{\epsilon}(x)<1$, then $\left\|x^{n}\right\| \rightarrow 0$ as $n \rightarrow \infty "$.

"Lemma 2.5 [2]: If $E$ is a real Banach algebra with cone $C$ and if $o \preccurlyeq u \leqslant c$ for each $o \preccurlyeq c$, therefore $u=o$."

"Lemma 2.6 [2]: Let $C$ be a cone and $a \preccurlyeq b+c$ for $c \epsilon C$, then $a \preccurlyeq b$.

A sub set $C$ of $E$ is called a algebra cone of $E$ if

1) $C$ non- empty closed and $\{0, e\} \subset C$ 
2) $\alpha a+\beta b \in C$ for all $\alpha, \beta>0$

3) $x . y \epsilon C$

4) $C \cap(-C)=\{0\} . "$

" We can define a preference ordering $\leqslant$ with respect to $C$ by $x \leqslant y$ iff $y-x \in C . x<y$ with stand for $x \leqslant y$ and $x \leqslant y$ the cone $C$ is called normal if there exist $N>0$ such that, for all $x, y \in E$

$0 \preccurlyeq x \preccurlyeq y \Rightarrow\|x\| \preccurlyeq N\|y\| . "$

Now, we define a new construction called $\Delta$ - ordered Banach algebra.

Definition 2.7: Let $X$ be a non-empty. A function $\Delta_{\lambda}:[0, \infty) \times X \times X \rightarrow E$ is called an $\Delta$ - metric on $X$ if

1) $\Delta(\lambda, x, y) \geqslant 0$ for $x, y \in X, \lambda \geq 0$

2) $x=y$ if and only if $\Delta(\lambda, \mathrm{x}, \mathrm{y})=\mathrm{o}$

3) $\Delta(\lambda, x, y)=\Delta(\lambda, y, x)$

4) $\Delta(\lambda, \mathrm{x}, \mathrm{y}) \preccurlyeq \Delta(\mu, y, a)$ for , $\mu>\lambda>0$ and $x, y, a \in X$

5) $) \Delta(\lambda+\mu, \mathrm{x}, \mathrm{y}) \preccurlyeq \Delta(\lambda, \mathrm{x}, \mathrm{y})+\Delta(\mu, y, a)$

The triple $(X, E, \Delta)$ is called $\Delta$ - ordered Banach algebra.

Example 2.8: Let $X$ be locally compact Housdorff space, $C(X)=\{f \mid f: X \rightarrow R$, continuous function $\}$, and $C^{+}(X)=\{f \in C(X): f(x) \geq 0$ for all $x \in X\}$, define multiplication in the natural way. Therefore $C(X)$ with supermom norm is ordered Banach algebra. It is obvious that $(C(x), X, \Delta)$ is $\Delta$-ordered Banach algebra where

$\Delta:[0, \infty) \times X \times X \rightarrow C(X)$ by $\Delta(\lambda, a, b)=\sup |f(a)-f(b)| e^{\lambda}$

\section{2- Topological structure on $\Delta$ - ordered Banach Algebra}

Definition 3.1: Let $(X, E, C)$ be $\Delta$-ordered Banach algebra. For all $x \in X$, for all $c>0$, the set $B_{\Delta}(\lambda, x, c)=$ $\{y \in X: \Delta(\lambda, x, y) \prec c\}$ is called $\Delta$-ball with and radius $c>0$ and admits $x$.

And put $\beta=\left\{B_{\Delta}(\lambda, x, c): x \in X\right.$, and $\left.c>0\right\}$.

Theorem 3.2: Let $(E, C)$ be ordered Banach algebra, then $(X, E, \Delta)$ is a Housdorff space.

Proof:- Let $(E, X, \Delta)$ be a $\Delta$ - ordered Banach algebra. Let $x, y \in X$ with $x \neq y, \lambda, \mu \geq 0$, we take $c=$ $\Delta(\lambda+\mu, x, y), U=B\left(\lambda, x, \frac{c}{2}\right), V=B\left(\mu, y, \frac{c}{2}\right)$.

Then $x \in U$ and $y \epsilon V$. We support $U \cap V \neq \varnothing$. There exist $a \epsilon U \cap V$.

But $\Delta(\lambda+\mu, x, a) \preccurlyeq \Delta(\lambda, x, a)+\Delta(\mu, y, a) \preccurlyeq \frac{c}{2}+\frac{c}{2}=c$.

That is $c \prec c$ and this contradiction.

Then, $(X, E, \Delta)$ is a Housdorff space. 
Definition 3.3: Let $(X, E, \Delta)$ be $a \Delta$-ordered Banach algebra. A sequence $\left\{x_{n}\right\}$ in $(X, \Delta)$ converges to a point $x$ if for every $c \in E$ with $c>0$, there exist a positive integer $N_{0}$ such that $\Delta\left(\lambda, x_{n}, x\right)<c$ for $n \geq N_{0}$, we denoted by $\lim _{n \rightarrow \infty} x_{n}=x\left(x_{n} \rightarrow x\right.$ as $\left.(n \rightarrow \infty)\right)$.

Definition 3.4: Let $(C, E, \Delta)$ be $a \Delta$-ordered Banach algebra. A sequence $\left\{x_{n}\right\}$ is said to be Cauchy sequence if for each $c>0$ there exists a positive integer $N_{0}$ such that $\Delta\left(\lambda, x_{n}, x_{m}\right) \prec c$ for all $n, m \geq N_{0}$.

Examples 3.5: Let $(X, C(X), \Delta)$ a $\Delta$ - ordered Banach algebra in example (3.2), take the set of rational numbers Q.

Define $\Delta=[0, \infty) \times X \times X \rightarrow C(X)$ is in example. Let $\left\{x_{t}\right\}$ be a sequence defined by $\alpha_{t}=\left(1+\frac{1}{t}\right)^{t}$. We note that $x_{t} \in \mathbb{Q}$ for each $t \in \mathbb{Z}$, note that $\Delta\left(\lambda, x_{t}, x_{k}\right)=\left|f\left(x_{t}\right)-f\left(x_{k}\right)\right| e^{-\lambda}$

$=\left|\left(1+\frac{1}{t}\right)^{t}-\left(1+\frac{1}{k}\right)^{k}\right| e^{\lambda}$ as $t, k \rightarrow \infty$

$\Delta\left(\lambda, x_{t}, x_{k}\right) \rightarrow 0$

That is for each $c>\theta$, there is $N_{0} \in Z^{+}$such that $\Delta\left(\lambda, a_{t}, a_{k}\right) \prec c$ for all $t, k \geq N_{0}$.

Thus, $\left\{a_{t}\right\}$ is a Cauchy sequence, but $a_{t} \rightarrow e$ as $\rightarrow \infty, e \notin \mathbb{Q}$. Hence, $\left\{a_{t}\right\}$ is not convergent.

Definition 3.7: Let $(X, E, \Delta)$ and $\left(X^{\prime}, E^{\prime}, \Delta^{\prime}\right)$ are $\Delta$ - ordered Banach algebra. A mapping $f: X \rightarrow X^{\prime}$ is said to be continuous at $x \in X$ when ever $\left\{x_{n}\right\}$ convergent to $x_{\text {, then }}\left\{f\left(x_{n}\right)\right\}$ is convergent to $f(x)$.

Definition 3.8: Let $(X, E, \Delta)$ be $\Delta$-ordered Banach algebra, $(X, E, \Delta)$ is called complete if for each Cauchy sequence is convergent in $X$.

Definition 3.9: Let $(X, E, \Delta)$ be $\Delta$-ordered Banach algebra. A map T: $X \rightarrow X$ is called Lipchitz if for all $c>0$, there exist a vector $N \epsilon C$ with $\sigma_{\epsilon}(N) \prec 1$ for each $x, y \in X$,

$\Delta\left(\lambda, \mathrm{T}_{a}, \mathrm{~T}_{b}\right) \leqslant \mathrm{N} . \Delta(\mu, a, b)$ for all $x, y \in X$ and $\lambda \leq \mu$

Example 3.10: Let $([0, \infty), C(X), \Delta)$ be a $\Delta$ - ordered Banach algebra. Define $T: X \rightarrow X$ as follows $T(a)=\frac{a}{2}$

$$
\begin{aligned}
& \Delta\left(\lambda, \mathrm{T}_{a}, \mathrm{~T}_{b}\right)=\sup \left|f\left(\mathrm{~T}_{a}\right)_{-} f\left(\mathrm{~T}_{b}\right)\right| e^{\lambda} \\
= & \sup |f \circ T(a)-f \circ T(b)| e^{\lambda}=\sup \left|f\left(\frac{a}{2}\right)-f\left(\frac{b}{2}\right)\right| e^{\lambda} \\
= & \frac{1}{2} \sup |f(a)-f(b)| e^{\lambda} \\
& \Delta(\mu, a, b)=\sup |f(a)-f(b)| e^{\mu}
\end{aligned}
$$

That is $T$ is a Lipschitz map in $X$

Definition 3.11: Let $(X, E, \Delta)$ be $\Delta$-ordered Banach algebra. A sequence $\left\{x_{t}\right\}$ is said to be $m$ - sequence if for all $m>0$, there exists $t \in x_{t}$ such that $x_{t}<m$ for all $n \geq t$.

Lemma 3.12: Let $(X, E, \Delta)$ be $\Delta$ - ordered Banach algebra. $\left\{m x_{t}\right\}$ is a $m$-sequence for all $c>0$ if the sequence $\left\{x_{t}\right\}$ is a $m$ - sequence in $C$.

Proof:- Suppose $\left\{x_{t}\right\}$ is a $m$ - sequence for all $c>0$, there exists $t \epsilon \mathbb{Z}^{+}$such that $x_{t} \prec c$ for $n>t$. For all $c>0$ , $m x_{t} \preccurlyeq m c$ by take $\frac{c}{m}=t$. 


\section{3- Main Results}

Definition 4.1: Let $(X, E, \Delta)$ be $\Delta$ - ordered Banach algebra. $T: X \rightarrow X$ holds the contradiction condition if $\Delta\left(\lambda, \mathrm{T}_{x}, \mathrm{~T}_{y}\right) \preccurlyeq t^{n} \Delta\left(\frac{\lambda}{2^{n}}, x_{1}, x_{0}\right)$

Theorem 4.2: Let $(X, E, \Delta)$ be $\Delta$ - ordered Banach algebra. Suppose $T: X \rightarrow X$ holds the $\Delta$-contradiction condition

$$
\Delta\left(\lambda, \mathrm{T}_{x}, \mathrm{~T}_{y}\right) \preccurlyeq m_{1} \Delta\left(\left(\frac{\lambda}{4}\right), x, \mathrm{~T}_{x}\right)+m_{2} \Delta\left(\left(\frac{\lambda}{4}\right), \mathrm{T}_{x}, y\right)+m_{3} \Delta\left(\left(\frac{\lambda}{4}\right), x, \mathrm{~T}_{y}\right)+m_{4} \Delta\left(\left(\frac{\lambda}{4}\right), y, \mathrm{~T}_{y}\right)
$$

where $0<\sum_{i=1}^{4} m_{i} \leq 1$, for $\mathrm{i}=1,2,3,4$ Then $T$ is a unique fixed point in $X$.

Proof: choose $x_{0} \epsilon X, x_{1}=T_{x_{0}}$ and $x_{n+1}=\mathrm{T}_{x_{n}}$

Take $0<m_{i} \leq 1$, for $i=1,2,3,4$

First we see,

$$
\begin{aligned}
& \Delta\left(\lambda, x_{n+1}, x_{n}\right)=\Delta\left(\lambda, \mathrm{T}_{x_{n}}, \mathrm{~T}_{x_{n-1}}\right) \leqslant m_{1} \Delta\left(\left(\frac{\lambda}{4}\right), x_{n}, \mathrm{~T}_{x_{n}}\right)+m_{2} \Delta\left(\left(\frac{\lambda}{4}\right), x_{n-1}, T_{x_{n}}\right)+m_{3} \Delta\left(\left(\frac{\lambda}{4}\right), x_{n}, \mathrm{~T}_{x_{n-1}}\right)+ \\
& m_{4} \Delta\left(\left(\frac{\lambda}{4}\right), x_{n-1}, \mathrm{~T}_{x_{n-1}}\right)+ \\
& \leqslant m_{1} \Delta\left(\left(\frac{\lambda}{4}\right), x_{n}, x_{n+1}\right)+m_{2} \Delta\left(\left(\frac{\lambda}{4}\right), x_{n-1}, x_{n+1}\right)+m_{3} \Delta\left(\left(\frac{\lambda}{4}\right), x_{n}, x_{n}\right)+m_{4} \Delta\left(\left(\frac{\lambda}{4}\right), x_{n-1}, x_{n}\right) \\
& \left.\leqslant m_{1} \Delta\left((\lambda / 4), x_{n}, x_{n+1}\right)+m_{2} \Delta\left(\left(\frac{\lambda}{4}\right), x_{n-1}, x_{n}\right)+m_{3} \Delta\left((\lambda / 4), x_{n}, x_{n+1}\right)+m_{4} \Delta\left(\left(\frac{\lambda}{4}\right), x_{n-1}, x_{n}\right)\right] \\
& \leqslant\left(m_{1}+m_{3}\right) \Delta\left(\left(\frac{\lambda}{4}\right), x_{n}, x_{n+1}\right)+\left(m_{2}+m_{4}\right)\left[\left(\Delta\left(\left(\frac{\lambda}{4}\right), x_{n}, x_{n-1}\right)\right]\right. \\
& \quad \leqslant t_{1} \Delta\left(\left(\frac{\lambda}{4}\right), x_{n}, x_{n+1}\right)+t_{2} \Delta\left(\left(\frac{\lambda}{4}\right), x_{n}, x_{n-1}\right) \\
& \ldots \ldots \ldots \ldots . . . \leqslant t_{1}^{n} \Delta\left(\left(\frac{\lambda}{2^{n}}\right), x_{0}, x_{1}\right)+t_{2}^{n} \Delta\left(\left(\frac{\lambda}{2^{n}}\right), x_{1}, x_{0}\right)
\end{aligned}
$$

if follows that

$$
\Delta\left(\lambda, x_{n+1}, x_{n}\right) \preccurlyeq t_{1}^{n} \Delta\left(\left(\frac{\lambda}{2^{n}}\right), x_{0}, x_{1}\right)+t_{2}^{n} \Delta\left(\left(\frac{\lambda}{2^{n}}\right), x_{1}, x_{0}\right)
$$

$$
\Delta\left(\lambda, x_{n+1}, \mathrm{x}_{n}\right)=\left(t_{1}^{n}+t_{2}^{n}\right) \Delta\left(\left(\frac{\lambda}{4}\right), x_{0}, x_{1}\right)
$$

Put $k=\left(t_{1}^{n}+t_{2}^{n}\right)$ ，

$\frac{1-\varepsilon}{1+\varepsilon} \leq|k| \leq \frac{1+\varepsilon}{1-\varepsilon}$. For $0<\epsilon<1$

It is clearly see that $\sigma_{\epsilon}(k) \prec 1$

$$
\begin{aligned}
& \Delta\left(\lambda, x_{n+1}, x_{n}\right) \leqslant k \Delta\left(\frac{\lambda}{2^{n-1}}, x_{n}, x_{n-1}\right)+\cdots+k^{n} \Delta\left(\frac{\lambda}{2^{n-1}}, x_{1}, x_{0}\right) \\
& \Delta\left(\lambda, x_{n}, x_{n+m}\right) \leqslant k^{m} \Delta\left(\frac{\lambda}{2^{n-1}}, x_{n}, x_{n-1}\right)+\cdots+k^{n+m} \Delta\left(\frac{\lambda}{2^{n-1}}, x_{1}, x_{0}\right)
\end{aligned}
$$


When $\mathrm{n}, m \rightarrow \infty$ we have $\lim _{n, m \rightarrow \infty} \Delta\left(\lambda, x_{n}, x_{n+m}\right)=0$

Thus $\left\{x_{n}\right\}$ is Cauchy sequence in $(X, E, \Delta)$

Since $(X, E, \Delta)$ is Banach algebra

That is $(X, E, \Delta)$ is complete.

Then $\left\{x_{n}\right\}$ is convergent to $x^{*} \in X$ such that $x_{n} \rightarrow x^{*}$

Next, we claim that $x^{*}$ is a fixed point of $T$

Actually,

$\Delta\left(\frac{\lambda}{4}, \mathrm{~T}_{x^{*}}, x^{*}\right) \leqslant \Delta\left(\lambda, \mathrm{T}_{x^{*}}, x^{*}\right) \preccurlyeq K\left[\Delta\left(\frac{\lambda}{2}, x^{*}, \mathrm{~T}_{x_{n}}\right)+\Delta\left(\frac{\lambda}{2}, \mathrm{~T}_{x_{n}}, \mathrm{~T}_{x^{*}}\right)\right]$

$=k \Delta\left(\frac{\lambda}{2}, x^{*}, x_{n+1}\right)+k \Delta\left(\frac{\lambda}{2}, \mathrm{~T}_{x_{n}}, \mathrm{~T}_{x^{*}}\right)$

$\preccurlyeq k\left[m_{1} \Delta\left(\frac{\lambda}{4}, x^{*}, \mathrm{~T}_{x^{*}}\right)+m_{2} \Delta\left(\frac{\lambda}{4}, x_{n+1}, \mathrm{~T}_{x^{*}}\right)\right.$

$\left.+m_{3} \Delta\left(\frac{\lambda}{4}, x^{*}, \mathrm{~T}_{x_{n+1}}\right)+m_{4} \Delta\left(\frac{\lambda}{4}, x_{n+1}, \mathrm{~T}_{x_{n+1}}\right)\right]+k \Delta\left(\frac{\lambda}{2}, x_{n+1}, x^{*}\right)$

$\preccurlyeq k m_{1} \Delta\left(\frac{\lambda}{4}, x^{*}, \mathrm{~T}_{x^{*}}\right)+k^{2} m_{2} \Delta\left(\frac{\lambda}{4} \lambda, x_{n+1}, T x^{*}\right)+k^{2} m_{2} \Delta\left(\frac{\lambda}{4} \lambda, x^{*}, x_{n+1}\right)+k m_{3} \Delta_{\lambda}\left(\frac{\lambda}{4} \lambda, x^{*}, x_{n+1}\right)+$ $\left.k^{2} m_{4} \Delta\left(\frac{\lambda}{4}, T x^{*}, x^{*}\right)+k^{2} m_{2} \Delta\left(\frac{\lambda}{4}, x^{*}, x_{n}\right)\right]+k \Delta\left(\frac{\lambda}{2}, x_{n+1}, x^{*}\right)$

$=\left(k m_{1}+k^{2} m_{4}\right) \Delta\left(\frac{\lambda}{4}, x^{*}, \mathrm{~T}_{x^{*}}\right)+\left(k^{2} m_{2}+k^{2} m_{4}\right) \Delta\left(\frac{\lambda}{4}, x_{n}, x^{*}\right)$

$+\left(k^{2} m_{2}+k m_{3}\right) \Delta\left(\frac{\lambda}{4}, x^{*}, x_{n+1}\right)+k \Delta\left(\frac{\lambda}{2}, x^{*}, x_{n+1}\right)$

then

$\left(1-k m_{1}-k^{2} m_{4}\right) \Delta\left(\frac{\lambda}{4}, x^{*}, \mathrm{~T}_{x^{*}}\right) \leqslant\left(k^{2} m_{2}+k^{2} m_{4}\right) \Delta\left(\frac{\lambda}{4}, x_{n}, x^{*}\right)+\left(k^{2} m_{2}+k m_{3}+k\right) \Delta\left(\frac{\lambda}{4}, x^{*}, x_{n+1}\right)+$ $k \Delta\left(\frac{\lambda}{2}, x^{*}, x_{n+1}\right)$

$\Delta\left(\frac{\lambda}{4}, x^{*}, \mathrm{~T}_{x^{*}}\right) \preccurlyeq \frac{\left(k^{2} m_{2}+k^{2} m_{4}\right) \Delta\left(\frac{\lambda}{4}, x_{n}, x^{*}\right)+\left(k^{2} m_{2}+k m_{3}+k\right) \Delta\left(\frac{\lambda}{4}, x^{*}, x_{n+1}\right)+k \Delta\left(\frac{\lambda}{2}, x^{*}, x_{n+1}\right)}{\left(1-k m_{1}-k^{2} m_{4}\right)} \leqslant c$

We can see easily $\Delta\left(\lambda, x^{*}, \mathrm{~T}_{x^{*}}\right)=0$ is the mapping $T$ which has a fixed point $x^{*}$

At last, for uniqueness, if there is $y^{*}$ other fixed point, then

$\Delta\left(\lambda, x^{*}, y^{*}\right)=\Delta\left(\lambda, \mathrm{T}_{x^{*}}, \mathrm{~T}_{y^{*}}\right)$

$\preccurlyeq m_{1} \Delta\left(\frac{\lambda}{4}, x^{*}, \mathrm{~T}_{x^{*}}\right)+m_{2} \Delta\left(\frac{\lambda}{4}, x^{*}, \mathrm{~T}_{y^{*}}\right)+m_{3} \Delta\left(\frac{\lambda}{4}, y^{*}, \mathrm{~T}_{x^{*}}\right)+m_{4} \Delta\left(\frac{\lambda}{4}, x^{*}, \mathrm{~T}_{y^{*}}\right)$ $\preccurlyeq m_{1} \Delta\left(\frac{\lambda}{4}, x^{*}, T x^{*}\right)+m_{2} \Delta\left(\frac{\lambda}{4}, x^{*}, y^{*}\right)+m_{4} \Delta\left(\frac{\lambda}{4}, y^{*}, x^{*}\right)+m_{3} \Delta\left(\frac{\lambda}{4}, y^{*}, \mathrm{~T}_{y^{*}}\right)$

$\preccurlyeq\left(m_{2}+m_{4}\right) \Delta\left(\lambda, x^{*}, y^{*}\right)$

Since $0<\left(m_{2}+m_{4}\right)<1$, we deduce from lemma that $x^{*}=y^{*}$ 
Definition 4.3: Let $(E, C)$ be ordered Banach algebra with algebra cone $C$. Take $\Phi$ be the set of all functions $\varphi: E^{3} \rightarrow E$ satisfying the following properties:

1) $\varphi(e, e, e)=c \preccurlyeq e$

2) Let $a, b \in E$ be such that if either $a \preccurlyeq \varphi(a, b, b)$ or $a \preccurlyeq \varphi(b, a, b)$ or $a \preccurlyeq \varphi(b, a, a)$

Definition 4.3: A self-mapping $T$ on $\Delta$ - ordered Banach algebra $(X, E, \Delta)$ is called $\varphi$-contraction, if there exists a map $\varphi \epsilon \Phi$ satisfy

$\Delta\left(\lambda, \mathrm{T}_{x}, \mathrm{~T}_{y}\right) \preccurlyeq \varphi\left(\Delta(\lambda, x, y), \Delta\left(\lambda, x, \mathrm{~T}_{x}\right), \Delta\left(\lambda, y, \mathrm{~T}_{y}\right)\right)$

Theorem 4.4: Let $(X, E, \Delta)$ be $\Delta$ - ordered Banach algebra and T a $\varphi$ - contraction. If there exists $\lambda>0$ such that for all $x \in X$.

$\Delta\left(\lambda, x_{0}, \mathrm{~T}_{x_{o}}\right)=\sup \left\{\Delta\left(\lambda, x, \mathrm{~T}_{x}\right): x \in X\right\}$, then $T$ has a unique fixed point

Proof: Suppose $x_{0} \neq \mathrm{T}_{x_{0}}$. We take $\quad x=x_{0}, \quad y=\mathrm{T}_{x_{0}} \quad$ in $\quad(5.1) . \quad$ then $\quad \Delta\left(\lambda, \mathrm{T}_{x_{0}}, \mathrm{~T}^{2}{ }_{x_{0}}\right) \leqslant$ $\varphi\left(\Delta\left(\lambda, x_{0}, \mathrm{~T}_{x_{0}}\right), \Delta\left(\lambda, x_{0}, \mathrm{~T}^{2} x_{o}\right), \Delta\left(\lambda, \mathrm{T}_{x_{o}}, \mathrm{~T}^{2}{ }_{x_{o}}\right)\right)$

Since $\Delta\left(\lambda, \mathrm{T}_{x_{0}}, \mathrm{~T}^{2}{ }_{x_{0}}\right) \preccurlyeq k \cdot\left[\Delta\left(\lambda, x_{0}, \mathrm{~T}_{x_{0}}\right)\right]$. But given that

$\Delta\left(\lambda, x_{0}, \mathrm{~T}_{x_{0}}\right)=\sup \left\{\Delta\left(\lambda, x, \mathrm{~T}_{x}\right): x \in X\right\}$

Hence $\mathrm{T}_{x_{0}}=x_{0}$

For uniqueness, let $y_{0}$ be other fixed point of $T$ that is $\mathrm{T}_{y_{o}}=y_{0}$

Now, $\Delta\left(\lambda, x_{0}, y_{0}\right)=$

$\preccurlyeq \varphi\left(\Delta_{\frac{\lambda}{3}}\left(x_{0}, y_{0}\right), \Delta_{\frac{\lambda}{3}}\left(x_{0}, \mathrm{~T}_{x_{0}}\right), \Delta_{\frac{\lambda}{3}}\left(y_{0}, \mathrm{~T}_{y_{0}}\right)\right)$

$\preccurlyeq \varphi\left(\Delta_{\frac{\lambda}{3}}\left(x_{0}, y_{0}\right), \Delta_{\frac{\lambda}{3}}\left(x_{0}, x_{0}\right), \Delta_{\frac{\lambda}{3}}\left(y_{0}, y_{0}\right)\right)$

$\preccurlyeq \varphi\left(\Delta\left(\frac{\lambda}{3}, x_{0}, y_{0}\right), 0,0\right)$

There for $\Delta_{\lambda}\left(\lambda, x_{0}, y_{0}\right) \preccurlyeq 0$ or $\Delta_{\lambda}\left(x_{0}, y_{0}\right)=0$. Implies $x_{0} \neq y_{0}$

That is the fixed point is unique and this complete the proof

Theorem 4.5: Let $S$ and $T$ be self-mapping on $\Delta$ - Banach algebra $(X, E, \Delta)$ satisfy the condition

$\Delta_{\lambda}\left(\lambda, \mathrm{T}_{x}, \mathrm{~S}_{y}\right) \leqslant \varphi\left(\left(\Delta(\lambda, x, y), \Delta\left(\lambda, x, \mathrm{~T}_{x}\right), \Delta\left(\lambda, y, \mathrm{~S}_{y}\right)\right) \quad\right.$ for all $x, y \in X$

If there exists $y \in X$ such that

$\Delta\left(\lambda, y, \mathrm{~T}_{y}\right) \preccurlyeq \Delta\left(\lambda, z, S_{z}\right)$

Then there exist a unique common fixed point of $S$ and $T$

Proof: Let $\mathrm{T}_{y_{0}}=x_{0}$, put $x=x_{0}, y=\mathrm{T}_{x_{0}}$, we obtain 
$\Delta_{\lambda}\left(\lambda, \mathrm{T}_{x_{0}}, S\left(\mathrm{~T}_{x_{0}}\right)\right) \leqslant \varphi\left(\Delta\left(\lambda, x_{0}, \mathrm{~T}_{x_{0}}\right), \Delta\left(\lambda, x_{0}, \mathrm{~T}_{x_{0}}\right), \Delta\left(\lambda, x_{0}, S\left(\mathrm{~T}_{x_{0}}\right)\right.\right.$

By (3) we get

$\Delta\left(\lambda, \mathrm{T}_{x_{0}} \cdot S\left(\mathrm{~T}_{x_{0}}\right)\right) \leqslant k \Delta\left(\lambda, x_{0}, \mathrm{~T}_{x_{0}}\right) \leqslant \Delta\left(\lambda, x_{0}, \mathrm{~T}_{x_{0}}\right)$

This contradict of (4.1)

To prove that $x_{0}$ is also a fixed point of $S$, let $\mathrm{S}_{x_{0}}=x_{0}$, therefore.

$\Delta\left(\lambda, x_{0}, \mathrm{~S}_{x_{0}}\right)=\Delta\left(\lambda, \mathrm{T}_{x_{0}}, \mathrm{~S}_{x_{0}}\right) \preccurlyeq \varphi\left[\Delta\left(\lambda, x_{0}, x_{0}\right), \Delta\left(\lambda, x_{0}, \mathrm{~T}_{x_{0}}\right), \Delta\left(\lambda, x_{0}, \mathrm{~S}_{x_{0}}\right)\right]$

Or $\Delta\left(\lambda, x_{0}, \mathrm{~S}_{x_{0}}\right) \leqslant \varphi\left(0,0, \Delta\left(\lambda, x_{0}, \mathrm{~S}_{x_{0}}\right)\right)$ that is $\Delta\left(\lambda, x_{0}, \mathrm{~S}_{x_{0}}\right) \leqslant 0$ or $\mathrm{S}_{x_{0}}=x_{0}$

For uniqueness, let $y_{0}$ be another fixed point of $S$ and $T$ that is

$\mathrm{T}_{y_{0}}=\mathrm{S}_{y_{0}}=y_{0}$, then

$\Delta\left(\lambda, x_{0}, y_{0}\right)=\Delta\left(\lambda, \mathrm{T}_{x_{0}}, \mathrm{~T}_{y_{0}}\right) \preccurlyeq \varphi\left[\left(\Delta\left(\lambda, x_{0}, y_{0}\right), \Delta\left(\lambda, x_{0}, \mathrm{~T}_{x_{0}}\right), \Delta\left(\lambda, y_{0}, \mathrm{~T}_{y_{0}}\right)\right]\right.$

Or $\Delta_{\lambda}\left(\lambda, x_{0}, y_{0}\right) \preccurlyeq \varphi\left(\Delta\left(\lambda, x_{0}, y_{0}\right), \Delta\left(\lambda, x_{0}, x_{0}\right), \Delta\left(\lambda, y_{0}, y_{0}\right)\right)$

$\preccurlyeq \varphi\left(\Delta\left(\lambda, x_{0}, y_{0}\right), 0,0\right)$

That is $\Delta\left(\lambda, x_{0}, y_{0}\right) \leqslant 0$ implies $x_{0}=y_{0}$.

Corollary 4.6: Let $S$ and $T$ be self-mapping of $\Delta$ - ordered Banach algebra $(X, E, \Delta)$ satisfying the following conditions:

1) There exists integer $n$ and $m$ such that

$\Delta\left(\lambda, \mathrm{T}^{n}{ }_{x}, \mathrm{~S}^{m}{ }_{y}\right) \preccurlyeq \varphi\left[\Delta(\lambda, x, y), \Delta\left(\lambda, x, \mathrm{~T}^{n}{ }_{x}\right), \Delta\left(\lambda, y, S^{m}{ }_{y}\right]\right.$ for some $\varphi \in \Phi$

2) If there exists a point $y \in X$ such that $\Delta\left(\lambda, y, \mathrm{~T}^{n}{ }_{x}\right) \preccurlyeq \Delta\left(\lambda, x, \mathrm{~S}^{m}{ }_{x}\right)$

Then there exists a unique common fixed point of $S$ and $T$

Theorem 4.7: Let $(X, E, \Delta)$ be a $\Delta$ - ordered Banach algebra such that

$\Delta\left(\lambda, \mathrm{T}_{x}, \mathrm{~T}_{y}\right) \preccurlyeq \min \left\{\lambda \Delta\left(\lambda, x, \mathrm{~T}_{x}\right), \mu \Delta\left(\mu, y, \mathrm{~T}_{y}\right)\right\}$

If there exists function $F$ defined by $F(x)=\lambda \Delta_{\lambda}\left(\lambda, x, \mathrm{~T}_{x}\right)$ for each $x \in X$ such that $F(x) \preccurlyeq F(\mathrm{~T}(x))$, then, $T$ has a unique fixed point

Proof: Suppose for some $x_{0}, x_{0} \neq \mathrm{T}_{x_{0}}$. Then $F\left(\mathrm{~T}_{x_{0}}\right)=\Delta\left(\lambda, \mathrm{T}_{x_{0}}, T\left(\mathrm{~T}\left(x_{0}\right)\right) \leqslant \min \left\{\lambda \Delta\left(\lambda, x_{0}, \mathrm{~T}_{x_{0}}\right), \mu \Delta\left(\mu, \mathrm{T}_{x_{0}}, \mathrm{~T}_{x_{0}}\right\}\right.\right.$ since $\Delta\left(\mathrm{T}_{x_{0}}, \mathrm{~T}_{x_{0}}\right)=\theta$

$\Delta\left(\lambda, \mathrm{T}_{x_{0}}, \mathrm{~T}\left(\mathrm{~T}\left(x_{0}\right)\right) \leqslant \lambda \Delta\left(\lambda, x_{0}, \mathrm{~T}_{x_{0}}\right)\right.$

$F\left(\mathrm{~T}_{x_{0}}\right) \preccurlyeq F\left(x_{0}\right)$ which is contradiction

Hence $\mathrm{T}_{x_{0}}=x_{0}$

For uniqueness, let $y$ be another point of $X$ different from $x_{0}$ such that $y_{0}=\mathrm{T}_{y_{0}}$, then 


$$
\begin{aligned}
& \Delta\left(\lambda, x_{0}, y_{0}\right)=\Delta\left(\lambda, \mathrm{T}_{x_{0}}, \mathrm{~T}_{y_{0}}\right) \preccurlyeq \min \left\{\lambda \Delta\left(\lambda, x_{0}, \mathrm{~T}_{x_{0}}\right), \lambda \Delta\left(\lambda, y_{0}, \mathrm{~T}_{y_{0}}\right)\right\} \\
& =\min \left\{\lambda \Delta\left(\lambda, x_{0}, x_{0}\right), \lambda \Delta\left(\lambda, y_{0}, y_{0}\right)\right\}=\min \{\theta, \theta\} \\
& \Delta\left(\lambda, x_{0}, y_{0}\right) \preccurlyeq \theta
\end{aligned}
$$

Hence $\Delta\left(\lambda, x_{0}, y_{0}\right) \preccurlyeq 0$ which implies that $\Delta\left(\lambda, x_{0}, y_{0}\right)=0$ or $y_{0}=x_{0}$

The proof is complete.

Theorem 4.8: Let $T$ be a self-map on a compact $\Delta$ - ordered Banach algebra $(E, A, C)$ satisfy Lipsctiz condition Then, $T$ has a unique fixed point.

Proof: Suppose $T$ satisfy Lipsctiz condition. Then, $T$ is a continuous map on $X$ we define a function from $X$ into $x$ as $F(x)=\Delta\left(\lambda, x, \mathrm{~T}_{x}\right)$ for all $x \in X$.

Since $T$ and $\Delta$ are continuous, it follow $F$ is continuous on $X$. Since $X$ is compact there exists a point $y \in X$ such that $F(y)=\inf \left\{\Delta\left(\lambda, x, \mathrm{~T}_{x}\right): x \in X\right\}$.

We support that $y \neq \mathrm{T}_{y}$.

Otherwise, that a fixed point by Lipscitiz condition

We have $\Delta\left(\lambda, \mathrm{T}_{y}, \mathrm{~T}^{2}{ }_{y}\right) \leqslant k \Delta\left(\mu, y, \mathrm{~T}_{y}\right) .0<\lambda \leq \mu$

So that $F\left(\mathrm{~T}_{y}\right) \preccurlyeq T(y)$ which contradiction.

Then, $y=\mathrm{T}_{y}$

Uniqueness follows from Lipscitz condition.

Proposition 4.9: Let $(X, E, \Delta)$ be a complete $\Delta$ - ordered Banach algebra. Assume that the mapping $\mathrm{T}: X \rightarrow X$ satisfy.

$\Delta\left(\lambda, \mathrm{T}^{n}{ }_{x}, \mathrm{~T}^{n}{ }_{y}\right) \leqslant k \Delta(\lambda, x, y)$, For each $x, y \in X$, for $n \in Z^{+}$, where $k$ a vector with is $\sigma_{\varepsilon}(k)<1$. Then, $T$ has a unique fixed point

Proof: $\mathrm{T}^{n}\left(\mathrm{~T}_{x^{*}}\right)=\mathrm{T}\left(\mathrm{T}^{n} x^{*}\right)=\mathrm{T}^{n} x^{*}=\mathrm{T}\left(\mathrm{T}^{n-1} x^{*}\right)=\mathrm{T}^{n-1}\left(x^{*}\right)=\cdots=\mathrm{T} x^{*}$.

So, $\mathrm{T} x^{*}$ is also has fixed point of $\mathrm{T}^{n}$ then $T x^{*}=x^{*}$

$x^{*}$ is a fixed point of $\mathrm{T}$.

Theorem 4.10: Let $(X, E, \Delta)$ be a compact $\Delta$ - ordered Banach algebra. Suppose the mapping satisfy $\Delta-$ lipshtiz condition in the following:

$\Delta\left(\lambda, \mathrm{T}_{x}, \mathrm{~T}_{y}\right) \leqslant k\left[\beta \Delta\left(\beta, \mathrm{T}_{x}, y\right)+\mu \Delta\left(\mu, \mathrm{T}_{y}, x\right)\right]$, for all $x, y \in X$, where $k$ is a vector with $k \epsilon(0,1)$. Then, $T$ has a unique fixed point in $X$. Another sequence $\left\{T_{x}^{t}\right\}$ converge to the fixed point.

Proof: choose $x_{0} \in X$ and set $x_{t}=T_{x}^{t}, t \geq 1$, we have for $t<m$

$\Delta\left(\lambda, x_{t+1}, x_{m}\right) \preccurlyeq \beta \Delta\left(\beta, x_{t}, x_{t+1}\right)+\mu \Delta\left(\mu, x_{t+1}, x_{m}\right)$ 


$$
\begin{aligned}
& \leqslant \beta \Delta\left(\beta, x_{t}, x_{t+1}\right)+\mu\left[\beta \Delta\left(\beta, x_{t+1}, x_{t+2}\right)+\mu \Delta\left(\mu, x_{t+2}, x_{m}\right)\right] \\
& \leqslant \beta \Delta\left(x_{t}, x_{t+1}\right)+\mu \beta \Delta\left(x_{t+1}, x_{t+2}\right)+\mu^{2}\left[\beta \Delta\left(x_{t+2}, x_{t+1}\right)+\mu \Delta\left(x_{t+3}, x_{m}\right)\right] \\
& \leqslant \beta \Delta\left(x_{t}, x_{t+1}\right)+\mu \beta \Delta\left(x_{t+1}, x_{t+2}\right)+\mu^{2} \beta \Delta\left(x_{t+1}, x_{t+2}\right)+\mu^{3} \beta \Delta\left(x_{t+3}, x_{t+1}\right)+\mu^{4} \Delta\left(x_{t+4}, x_{m}\right)
\end{aligned}
$$

$\preccurlyeq \beta\left[\Delta\left(\lambda, x_{t}, x_{t+1}\right)+\mu \Delta\left(\lambda, x_{t+1}, x_{t+2}\right)+\mu^{2} \Delta\left(\lambda, x_{t+2}, x_{t+3}\right)+\ldots+\right.$

$\left.\mu^{n} \Delta\left(\lambda, x_{1}, x_{0}\right)\right]+\mu^{n}\left[\Delta\left(\lambda, x_{t+m}, x_{m}\right)\right]$

$\preccurlyeq \beta\left[k^{t} \Delta\left(\lambda, x_{1}, x_{0}\right)+\mu k^{t+1} \Delta\left(\lambda, x_{1}, x_{0}\right)+\cdots+\mu^{m} k^{t+m} \Delta\left(\lambda, x_{1}, x_{0}\right)\right.$

$+\mu^{t+1}\left[k^{t+m+1} \Delta\left(\lambda, x_{1}, x_{0}\right)\right]$

$\preccurlyeq \beta k^{t}\left[1+\mu k+\cdots+\mu^{m} k^{m}\right] \Delta\left(\lambda, x_{1}, x_{0}\right)+\mu^{t} k^{t+m} \Delta\left(\lambda, x_{1}, x_{0}\right)$

$\preccurlyeq \beta k^{t}\left[\sum_{i=1}^{m} \mu^{i} k^{i}\right] \Delta\left(\lambda, x_{1}, x_{0}\right)+\mu^{m+1} k^{t+m} \Delta\left(\lambda, x_{1}, x_{0}\right)$

$\preccurlyeq \beta k^{t}\left[\sum_{i=1}^{m+1} \mu^{i} k^{i} \Delta\left(\lambda, x_{1}, x_{0}\right)\right]$

$\preccurlyeq \beta k^{t}\left[\sum_{i=1}^{\infty} \mu^{i} k^{i}\right] \Delta\left(\lambda, x_{1}, x_{0}\right)$

$\preccurlyeq \beta k^{t}\left[\sum_{i=1}^{0} \mu^{i} k^{i}\right] \Delta\left(\lambda, x_{1}, x_{0}\right)$

$\preccurlyeq \beta k^{t}(e-\mu k)^{-1} \Delta\left(\lambda, x_{1}, x_{0}\right)$

$\left\|\Delta\left(\lambda, x_{n+1}, x_{m}\right)\right\| \leq\left\|\beta k^{t}\right\| \cdot\left\|(e-\mu k)^{-1}\right\| \cdot\left\|\Delta\left(\lambda, x_{1}, x_{0}\right)\right\|$

Since $\left\|k^{n}\right\| \rightarrow 0$ as $n \rightarrow \infty$, where $\left\|\Delta\left(\lambda, x_{n}, x_{m}\right)\right\| \rightarrow 0$ as $n \rightarrow \infty$

Which implies $\Delta\left(\lambda, x_{t}, x_{m}\right) \rightarrow 0$ as $(t, m \rightarrow 0)$

Hence, $\left\{x_{t}\right\}$ is a Cauchy sequence. since $X$ is complete, there exists $x^{*} \in X$ such that $x_{t} \rightarrow x^{*}$ as $n \rightarrow \infty$, therefore $\lim \Delta\left(\lambda, T_{x^{*}}, x^{*}\right) \preccurlyeq k\left[\beta \Delta\left(\lambda, T_{x_{t}}, T_{x^{*}}\right)+\mu \Delta\left(\lambda, T_{x^{*}}, x_{t}\right)\right]$

$\preccurlyeq \beta k\left[\Delta\left(\lambda, x_{t}, x^{*}\right)+\Delta\left(\lambda, x^{*}, x_{t+1}\right)\right]+\mu \Delta\left(\lambda, T_{x^{*}}, x_{t}\right)$

$\left\|\Delta\left(\lambda, T_{x^{*}}, x^{*}\right)\right\|$

$\leq\|\lambda\| \cdot\|k\| \cdot\left[\left\|\Delta\left(\lambda, x_{t}, x^{*}\right)\right\|+\left\|\Delta\left(\lambda, x^{*}, x_{t+1}\right)\right\|\right]+\|\mu\| \cdot\left\|\Delta\left(\lambda, x_{t}, x^{*}\right)\right\|$

Which implies $T_{x^{*}}=x^{*}$ and so $x^{*}$ is fixed point

To prove uniqueness, let $b$ be another fixed point of $T$.

Then $\Delta\left(\lambda, x^{*}, b\right)=\Delta\left(\lambda, T_{x^{*}}, T_{b}\right) \leq k\left[\beta \Delta\left(\lambda, T_{x^{*}}, b\right)+\mu \Delta\left(\lambda, T_{b}, x^{*}\right)\right]$

$=k\left[\beta \Delta\left(\lambda, x^{*}, b\right)+\mu \Delta\left(\lambda, b, x^{*}\right)\right]=k[\lambda+m] \Delta\left(\lambda, x^{*}, b\right)$ 
Then, $\left[1-k(\beta+\mu] \Delta\left(\lambda, x^{*}, b\right) \leq 0\right.$.

Since $k \epsilon(0,1)$ and $\beta, \mu>0 \Rightarrow \Delta\left(\lambda, x^{*}, b\right)=0$ so $x^{*}=b$

The proof is complete.

\section{5-Conclusion}

In this paper, we introduce a new concept which is called $\Delta$ - ordered Banach algebra. Also, we define lipshtiz condition in this pace, $\varphi$ - contraction, $\Delta-$ contraction and $\Delta-$ lipshtiz condition. In the new work, we prove fixed point theorems satisfying these maps in $\Delta$ - ordered Banach algebra. Our conditions and results are new in comparison with those of the results of cone metric space. These results can be extended to other spaces.

\section{References}

1. Akbar Zada, Rahim Shah and Shahid Saifullah,"Some Fixed Point Theorems in Multiplicative Cone b- Matric Spaces", Journal of Analysis \& Number Theory, 5, No. 1, 35-39, (2017).

2. B. Y.Hussein. and S. F.Hassan,"Some properties of Characterization on ordered Banach algebra",Ms.C. thesis ,Al-Qadisiyah university,2017.

3. Arun Gary, Zaheer K. Ansari," Cone Metric Spaces and Fixed Point Theorems of T-Contractive Mapping", International journal of Mathematics and Statistics Invention (IJMSI), Vol.2, Iss. 2, PP. 72-76, (2014)

4. D. Dey and M.Saha," Partial Cone Metric Space and Some Fixed Point Theorems", TWMS J.App. Math. $U_{3}$, N.1, PP. 1-9, (2013).

5. Dhage, B.C.,"A study of some fixed point theorem", Ph. Thesis, Marath Wada University, Aurangabad, (1984).

6. Gahler, S., " 2- Metriche Raume and Ihre Topologisch Structure", Mathematiche Nachrichten, 26, 115-148, (1963).

7. G. S. Sulugq and B. P. Tripathi, "Some Common Fixed Point Theorems of Generalized Contractive Mappings in Cone Metric Spaces", Functional Analysis Approximation and Computation, 5:1, 33-41, (2013).

8. Hao Liu and Shaoman XU, "Conemetric Spaces with Banach Algebras and Fixed Point Theorems of Generalized Lipschitz Mapping, Springre, Fixed Point and Application, 320, (2013).

9. Huang, L., G., \& Zhang X, "Cone Metric Space and Fixed Point Theorem for Contractive Mapping", Journal of Mathematical Analysis and Applications, 332, (1468-1476).

10. H. K Nashine and I. Altun, "A Common Fixed Point Theorem on Ordered Metric Spaces", Bulletin of The Iranian Mathematical Society, Vol. 38 No. 4, PP. 925- 934, (2012).

11. H. Liu and S. Xu," Cone Metric Spaces with Banach Algebra and Fixed Point Theorems of Generalized Lipchitz Mappings," Fixed Point Theory and Applications, 320. Dio:10.118611687-1812-320 (2013).

12. H. Rahimi and G. Soleimani Rad, "Fixed Point Theorems Under C. Distance in Ordered Cone Metric Space", Int. J. Industrial Mathematics, Vol. 6, No. 2, Article IDISIM-00253, 9 Page, (2014).

13. Jerolina Fernardcz, Sompob Sale, Kalpana Saxena, Neeraj Malviya and Poom Kuman, " The A- Cone Metric Space Over Banach Algebra with Applications ", Fernardczct. at. Cogent Mathematics, 4:1828690, (2017). 
14. K. P. R Sastry, Ch-Srinivasa Rao, A. Chandra Sekhar and M. Balaiah,"A Fixed Point Theorem in Cone Metric Spaces Under Weak Contractions", Gen. Math. Notes, Vol. 7, No. 2, PP.38-44, (2011).

15. K. Ha, Y. J. Choand Awhile, "Striclly Convex and 2- Convex 2- Normd Spacy." Mathematica Japonica, 33, 375-384, (1988).

16. L. G. Huang and X. Zhand, " Cone Metric Spaces and Fixed Point Theorems for Contractive Mappings ", Journal of Mathematics Analysis and Applications, 332, 1468-1476, (2007).

17. Mustafa and B. Sims, " A New Approach to Generalized Metris spaces", Journal of Nonlinear and Convex Analysis, 7, 289- 297, (2006).

18. Sedghi S., Shobe, N., \& Aliouche, A. " A Generalization of Fixed Point Theorems in S- Metric Spaces ", Mathematichki Vesnik, 64, 258-266, (2012).

19. R. De Jong, "Ordered Banach Algebras", Ms.C. Thesis, Leiden University. Holland, 2010.

20. Sedghi S., Shobe, N., \& Zhou, H., "A Common Fixed Point Theorem in D*- Metric Space", Fixed Point Theory and Applications, 29, 631-636, (2007).

21. Sevng Hyun Kim and Byvng Soo Lee, "Generalized Fixed Point Theorems in Cone Metric Spaces", Korean J. Math 20, No. 3, PP.353-360, (2012).

22. S. K. Tiwari and R. P. Dubey, "Cone Metric Spaces and Fixed Point Theorems for Generalized Y. Reich and T Rhoadg Contractive Mapping", Asin Journal of Mathematics and Applications Article Idama 0077, 9 Page, vol. (2013).

23. V. H. Badshah, P. Bhagatand S. Shukla, "Some Fixed Point Theorems for $\alpha-\phi$ - Outmetric Mapping in 2Metric Spaces", Scientific Publications of State University of Novipazar, Ser. A: ppl. Math. INF or M. and Mech, Vol 802, 181-199, (2016).

24. Zhenhua Ma, Lining Jiang, and Hongkaisun, " $C^{*}$ - Algebra- Valued Metric Spaces and Related Fixed Point Theorems", Ma et al. Fixed Point and Applications Springer: 206., (2014).

25. Yan Han, Shaoyuan Yu, " New Common Fixed Point Results for Four Maps on Cone Metric Spaces" Applied Mathematics, 2, 1114-1118,(2011). 